\title{
Erratum to: Herbal decoctosome is a novel form of medicine
}

\author{
Xiaoyun $\mathrm{Li}^{1 \dagger}$, Zhu Liang ${ }^{1 \dagger}$, Jianchao $\mathrm{Du}^{1 \dagger}$, Zhiqing Wang ${ }^{1 \dagger}$, Song Mei ${ }^{1}$, Zhiqing $\mathrm{Li}^{1}$, Yan Zhao ${ }^{1}$, \\ Dandan Zhao ${ }^{1}$, Yiming Ma ${ }^{1}$, Jun $\mathrm{Ye}^{1}$, Jiantao Xu${ }^{1}$, Yu Zhao ${ }^{1}$, Jiahui Chang ${ }^{1}$, Yuhao Qin ${ }^{1}$, \\ Lanlan $\mathrm{Yu}^{2}$, Chenxuan Wang ${ }^{2 *} \&$ Chengyu Jiang ${ }^{1^{*}}$ \\ ${ }^{1}$ State Key Laboratory of Medical Molecular Biology, Institute of Basic Medical Sciences, Chinese Academy of Medical Sciences, Department \\ of Biochemistry, Peking Union Medical College, Beijing 100005, China; \\ ${ }^{2}$ Institute of Basic Medical Sciences, Chinese Academy of Medical Sciences, Department of Biophysics and Structural Biology, Peking Union \\ Medical College, Beijing 100005, China
}

Received June 3, 2020; accepted June 17, 2020; published online July 8, 2020

Erratum to: Sci China Life, 2019, 62: 333-348, https://doi.org/10.1007/s11427-018-9508-0

Citation: Li, X., Liang, Z., Du, J., Wang, Z., Mei, S., Li, Z., Zhao, Y., Zhao, D., Ma, Y., Ye, J., et al. (2020). Erratum to: Herbal decoctosome is a novel form of medicine. Sci China Life Sci 63, 1428-1428. https://doi.org/10.1007/s11427-020-1760-4

Following the published article, we noticed an error duplication in Figure 5G "control" and "PGY-6" that was introduced during the revised process, with an attempt to replace it with higher-resolution images. Here we provide the original data in the first submitted manuscript (Figure 5G):

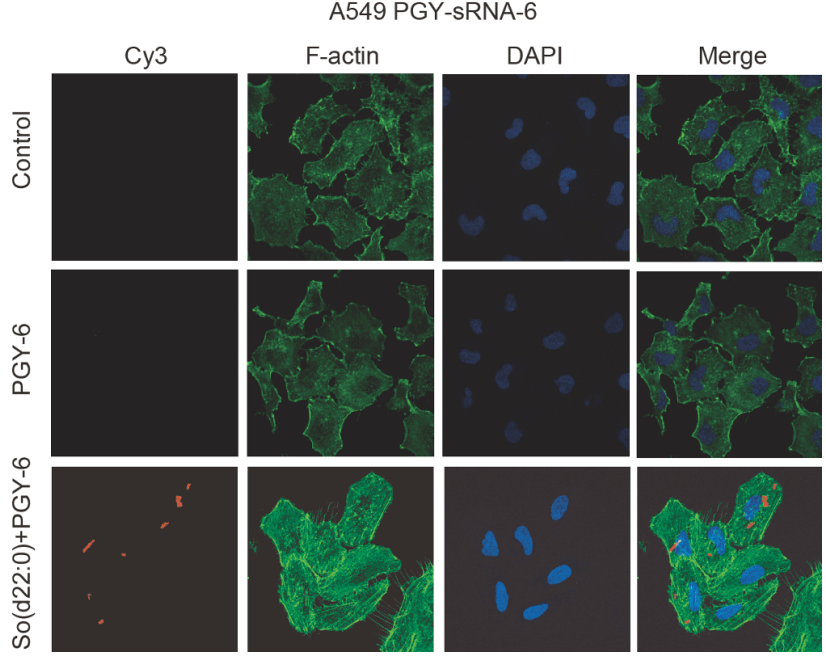

Figure 5 Bencaosomes exhibit medical effects in vitro. G, The localization of PGY-sRNA-6 in A549 cells was visualized by confocal microscopy. Cy3conjugated PGY-sRNA-6 (red), Alexa Flour 488 staining for F-actin (green), DAPI nuclear staining (blue) and merged images are indicated. A549 cells were collected after incubation with or without PGY-sRNA-6 or bencaosome for $6 \mathrm{~h}$.

The online version of the original article can be found at https://doi.org/10.1007/s11427-018-9508-0

$\dagger$ Contributed equally to this work

*Corresponding authors (Chengyu Jiang, email: jiang@pumc.edu.cn; Chenxuan Wang, email: wangcx@ibms.pumc.ed.cn) 